\title{
Pyroelectric and Dilatometric Studies on the Low Temperature Ferroelectric Phase Transition of $\left(\mathrm{CH}_{3} \mathrm{NH}_{3}\right)_{5} \mathrm{Bi}_{2} \mathrm{Br}_{11}$
}

\author{
J. DzIEDZIC ${ }^{a}$, J. Mróz ${ }^{a}$ AND R. JAKUBAS ${ }^{b}$ \\ ${ }^{a}$ Institute of Physics, Wrocław University of Technology \\ Wybrzeże Wyspiańskiego 27, 50-370 Wrocław, Poland \\ ${ }^{b}$ Faculty of Chemistry, University of Wrocław \\ Joliot-Curie 14, 50-383 Wrocław, Poland
}

(Received March 7, 2005)

\begin{abstract}
The results of the linear thermal expansion studies on $\left(\mathrm{CH}_{3} \mathrm{NH}_{3}\right)_{5} \mathrm{Bi}_{2} \mathrm{Br}_{11}$ single crystals in the temperature range $60-340 \mathrm{~K}$ are presented. The thermal anomaly in the vicinity of $77 \mathrm{~K}$ is assigned to the phase transition classified as close to first order type. The pyroelectric measurements of $\left(\mathrm{CH}_{3} \mathrm{NH}_{3}\right)_{5} \mathrm{Bi}_{2} \mathrm{Br}_{11}$ were carried out in the range of $45-320 \mathrm{~K}$ along the $a$ - and $c$-axis. The step-like change in the spontaneous polarization along the $a$-axis at $77 \mathrm{~K}$ indicates a discontinuous character of ferroelectric transition.
\end{abstract}

PACS numbers: $77.80 . \mathrm{Bh}$

\section{Introduction}

Recently the alkylammonium halogenoantimonates(III) and halogenobismuthates(III) of the general formula $\mathrm{R}_{a} \mathrm{M}_{b} \mathrm{X}_{3 b+a}$ (where $\mathrm{R}$ denotes organic cations, $\mathrm{M}-\mathrm{Sb}, \mathrm{Bi}$, and $\mathrm{X}-\mathrm{Cl}, \mathrm{Br}, \mathrm{I})$ evoke much interest. It was found that numerous compounds of this family underwent multiple structural phase transitions (PTs) [1-3]. The salts with the $\mathrm{R}_{3} \mathrm{M}_{2} \mathrm{X}_{9}$ or $\mathrm{R}_{5} \mathrm{M}_{2} \mathrm{X}_{11}$ compositions appeared to be specially interesting since they frequently demonstrate ferroelectric properties. The crystal structure of the $\mathrm{R}_{3} \mathrm{M}_{2} \mathrm{X}_{9}$ subclass was characterized by three types of anionic form: (1) zigzag double chains [4], (2) two-dimensional layers of the $\mathrm{MX}_{6}$ octahedra [5], and (3) by isolated $\mathrm{M}_{2} \mathrm{X}_{9}^{3-}$ units [6]. It was shown that polar 
properties are observed only for the salts built up of the layer anionic sublattice and small-sized cations, like $\mathrm{CH}_{3} \mathrm{NH}_{3}^{+},\left(\mathrm{CH}_{3}\right)_{2} \mathrm{NH}_{2}^{+}$, and $\left(\mathrm{CH}_{3}\right)_{3} \mathrm{NH}^{+}$[7-10]. The second subclass $-\mathrm{R}_{5} \mathrm{M}_{2} \mathrm{X}_{11}$ - was characterized exclusively by isolated $\mathrm{M}_{2} \mathrm{X}_{11}^{5-}$ units. There are only three known salts crystallizing with composition like this: $\left(\mathrm{CH}_{3} \mathrm{NH}_{3}\right)_{5} \mathrm{Bi}_{2} \mathrm{Br}_{11}$ [11], $\left(\mathrm{CH}_{3} \mathrm{NH}_{3}\right)_{5} \mathrm{Bi}_{2} \mathrm{Cl}_{11}$ [12], and $\left(\mathrm{C}_{5} \mathrm{H}_{5} \mathrm{NH}\right)_{5} \mathrm{Bi}_{2} \mathrm{Br}_{11}$ [13]. All of them exhibited the ferroelectric properties. The origin of the ferroelectricity of both subclasses was attributed to dynamical ordering of dipolar organic cations.

$\left(\mathrm{CH}_{3} \mathrm{NH}_{3}\right)_{5} \mathrm{Bi}_{2} \mathrm{Br}_{11}$ (MAPBB) at $311.5 \mathrm{~K}$ undergoes a ferroelectric phase transition of the $m m m F m m 2$ type (from $P c a b$ to $P c a 2_{1}$ space group) [14]. The dielectric dispersion [15] and the Raman scattering [16] studies indicate the "orderdisorder" mechanism of the phase transformation. Another phase transition is manifested by a nonlinear increase in spontaneous polarization and dielectric anomaly in the vicinity of $77 \mathrm{~K} \mathrm{[17].} \mathrm{The} \mathrm{character} \mathrm{of} \mathrm{this} \mathrm{phase} \mathrm{transition} \mathrm{is}$ still unclear and the symmetry (space group) of the lowest temperature phase is unknown. Nevertheless, the dynamics of the methylammonium cations is expected to contribute both to the paraelectric-ferroelectric transition at $311 \mathrm{~K}$ and the phase transition at $77 \mathrm{~K}$.

The linear thermal expansion studies on the MAPBB crystals have been limited to the high temperature ferroelectric transition $[18,19]$. To throw more light on the character of the phase transition at $77 \mathrm{~K}$ and the linear thermal expansion in the wide temperature region of MAPBB we decided to perform the dilatometric studies of the compound in the temperature range $350-60 \mathrm{~K}$. This paper also contains new pyroelectric measurements on MAPBB along three crystallographic directions (the $a-, b$-, and $c$-axis of the orthorhombic system) from $315 \mathrm{~K}$ to $30 \mathrm{~K}$, with a special inspection of the lowest temperature phase transition at $77 \mathrm{~K}$.

\section{Experimental procedure}

Single crystals of MAPBB were obtained in the reaction of $(\mathrm{BiO})_{2} \mathrm{CO}_{3}$ with $\mathrm{CH}_{3} \mathrm{NH}_{3} \mathrm{Br}$ in concentrated $\mathrm{HBr}$. The synthesized compound was purified by repeated crystallizations. Yellow and transparent single crystals were grown at room temperature and had the form of orthorhombic prism. Samples with three different orientations (plates perpendicular to the $a-, b$-, and $c$-axis) were prepared to study the pyroelectric and dilatometric properties.

The linear thermal expansion measurements in the temperature range 60$340 \mathrm{~K}$ were made with a capacitance dilatometer. Errors in $\Delta L / L_{0}$ values were generally smaller than $1 \%$.

The temperature dependence of the spontaneous polarization and pyroelectric coefficient were carried out by using the method of an integration of the pyroelectric charge (with an electrometer UNITRA 219-type). A detailed description of this method is given by Dziedzic and Stolecki in Ref. [20]. The spontaneous polarization $\left(P_{\mathrm{s}}\right)$ and the pyroelectric coefficient $(\gamma)$ measurements are accurate to within $15 \%$ and $10 \%$, respectively. 


\section{Results and discussion}

\subsection{Dilatometric measurements}

Figures 1-3 show results of the linear thermal expansion $\left(\Delta L / L_{0}\right.$, where $L_{0}$ is the length of the sample at room temperature for the corresponding axes) and the linear thermal expansion coefficient $\left(\alpha=\partial\left(\Delta L / L_{0}\right) / \partial T\right)$ as a function of temperature measured along the $a-, b$-, and $c$-axis (the directions presented correspond to those taken for the room temperature ferroelectric orthorhombic phase) during the cooling cycles. The linear thermal expansion for all axes of MAPBB in the vicinity of the paraelectric-ferroelectric phase transition is similar to that presented by Jakubas and Pykacz [18]. The $\alpha_{a}$ coefficient reveals a clear anomaly in the vicinity of the paraelectric-ferroelectric transition at $311.5 \mathrm{~K}$ and a small diffused anomaly in the vicinity of $220 \mathrm{~K}$. It should be noted that the expected phase transition at $77 \mathrm{~K}$ is rather hardly visible along the $a$-axis. The $\alpha_{a}$ coefficient changes from $2 \times 10^{-5} \mathrm{~K}^{-1}$ to about $4 \times 10^{-5} \mathrm{~K}^{-1}$ in the range 70-220 K.

Quite unusual behaviour of the linear thermal expansion is found along the $b$-axis. Below $311 \mathrm{~K}$ the MAPBB is characterized by a negative coefficient $\alpha_{b}$
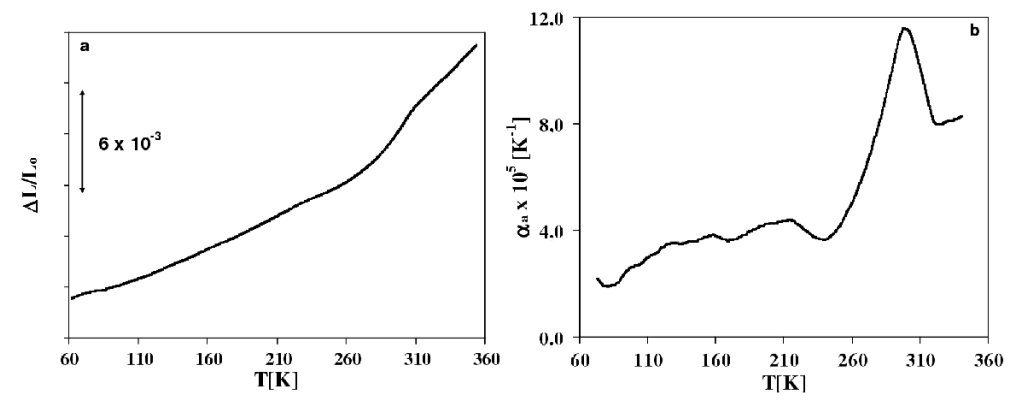

Fig. 1. (a) Temperature dependence of the linear thermal expansion and (b) the linear thermal expansion coefficient for MAPBB along the $a$-axis on cooling.
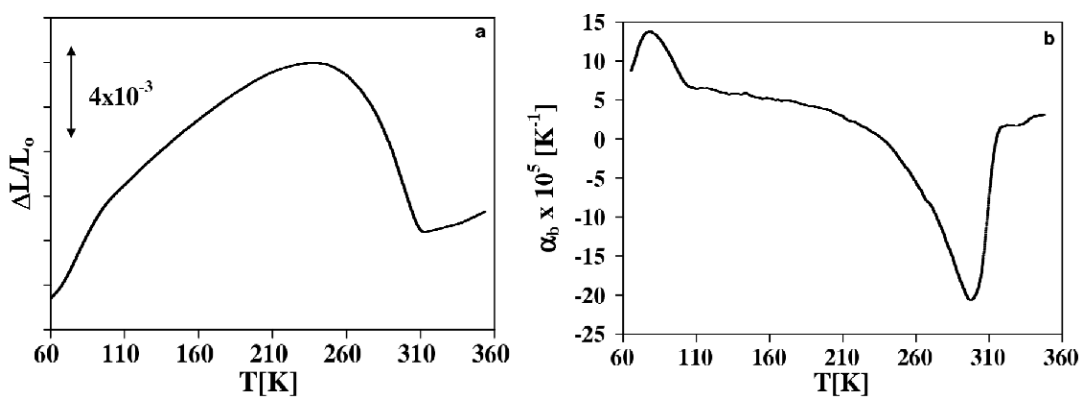

Fig. 2. The same as in Fig. 1 but along the $b$-axis. 

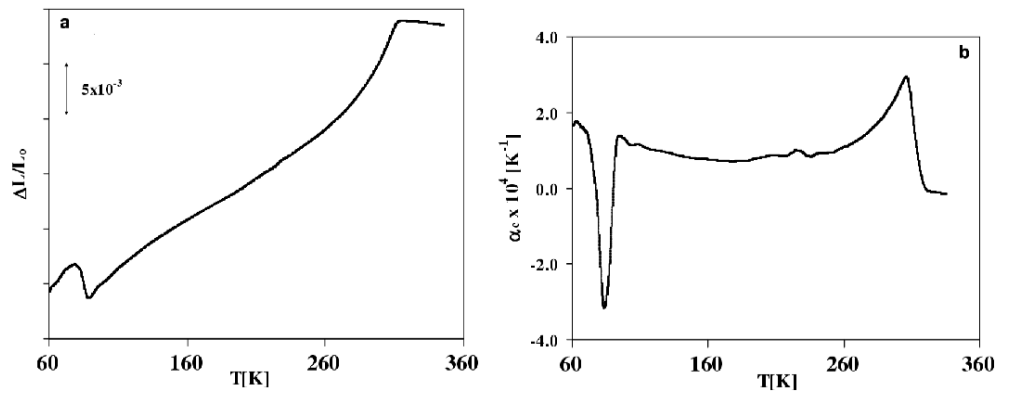

Fig. 3. The same as in Fig. 1 but along the $c$-axis.

with $\alpha_{b \max }=2 \times 10^{-4} \mathrm{~K}^{-1}$. At $240 \mathrm{~K}$ the thermal expansion displays a change of sign of the $\alpha$ coefficient. On further cooling a visible anomaly in dilation of the crystal is observed close to $77 \mathrm{~K}$ accompanied by a clear peak on the $\alpha_{b}$ versus temperature curve.

The most interesting properties of the linear thermal expansion of MAPBB over the low temperature region were found along the polar $c$-axis. In the wide temperature region (100-260 K) the $\alpha_{c}$ coefficient maintains a constant value of about $1 \times 10^{-4} \mathrm{~K}^{-1}$. Close to $80 \mathrm{~K}$ a rapid expansion of the sample $\left(-\Delta L / L_{0}\right)$ of the order of $3 \times 10^{-3}$ is observed due to the structural phase transitions between two polar phases. The transition is accompanied by an appearance of a significant $\alpha_{c}$ coefficient $\left(\approx 3 \times 10^{-4} \mathrm{~K}^{-1}\right)$. It should be underlined that the observed phase transition in MAPBB at $77 \mathrm{~K}$ has some features of the first order one. One can conclude that the $77 \mathrm{~K}$ phase transition reveals a mixed character and it is rather close to the first order type.

The changes in the volume of the crystals $\left(\Delta V / V_{0}\right)$ and the volume thermal expansion coefficient $\left(\alpha_{V}\right)$ in the studied temperature range are illustrated in Fig. 4. The both phase transitions are evidently shown on the $\Delta V / V_{0}$ versus temperature curve. It is clearly seen that the $\alpha_{V}$ coefficient accompanying the transition at $77 \mathrm{~K}$ is distinctly larger than that for the paraelectric-ferroelectric
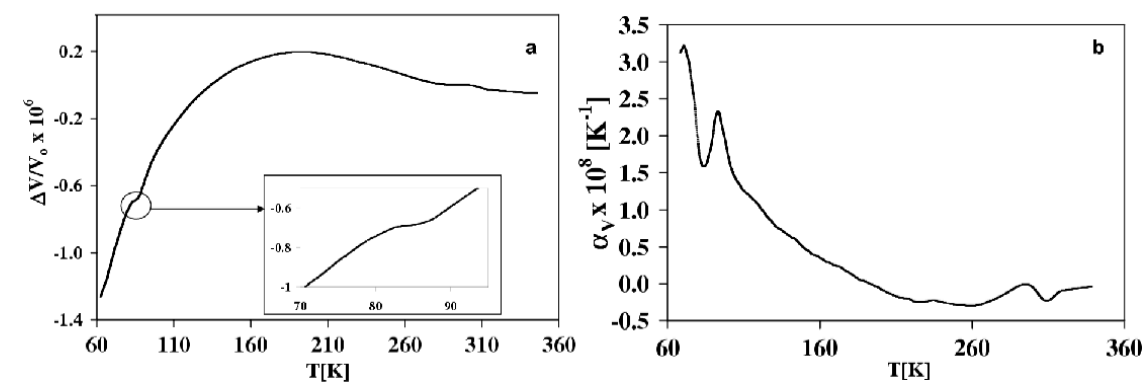

Fig. 4. (a) Temperature dependence of the relative $\Delta V / V_{0}$ and (b) the volume thermal expansion coefficient for MAPBB in a wide temperature range. The inset in part (a) shows an anomaly of $\Delta V / V_{0}$ in the vicinity of $77 \mathrm{~K}$. 
transition $(311.5 \mathrm{~K})$. The dilatometric response of MAPBB shows that the phase transition at $77 \mathrm{~K}$ is featured as a discontinuous one in contrast to the clear second order transition at $311 \mathrm{~K}$. It is interesting to notice that the $\Delta V / V_{0}$ versus temperature curve exhibits a maximum at about 200-190 K which corresponds to the minimum visible on the $P_{\mathrm{s}}(T)$ curve (see also Fig. 5). Such a behaviour can be explained by interactions of dipolar units (methylammonium cations). An increase in the relative volume of the unit cell of the crystal leads to an increase in the distances between interacting dipoles contributing to the spontaneous polarization. As a result of this fact a continuous reduction of spontaneous polarization is observed below 250-260 K.

\subsection{Pyroelectric measurements}

The temperature dependence of the spontaneous polarization $\left(P_{\mathrm{s}}\right)$ along the polar $c$-axis in a wide temperature region is shown in Fig. 5 . The pyroelectric response in the vicinity of the paraelectric-ferroelectric phase transition is similar to that reported in Ref. [17]. Nevertheless, a small but clearly visible anomaly as a jump in the $\Delta P_{\mathrm{s}}$ value (see the inset of Fig. 5) is recorded. Besides, it turns out that the samples of virgin single crystals of MAPBB have a tendency to exist in a single domain state without any dc pooling. In our experiment before pyroelectric measurements the sample was pooled in a relatively small external dc electric field of the order of $10 \mathrm{~V} \mathrm{~cm}^{-1}$. The dc electric field was switched in an opposite direction to the spontaneous polarization vector of a singledomain sample (not polarized before). It was shown that an increase in the pooling dc electric field led to a larger step-like anomaly on the $P_{\mathrm{s}}$ vs. temperature curve at $77 \mathrm{~K}$. A detailed account of the electric field effect on the low temperature phase transition will be published elsewhere.

The most interesting behaviour of $P_{\mathrm{s}}$ is observed close to the phase transition at $77 \mathrm{~K}$. A proper inspection of the pyroelectric coefficient $(\gamma(T))$ reveals two peaks situated closely to each other. It indicates the complex mechanism of this phase transition. The higher temperature peak on the $\gamma_{c}(T)$ curve nearly $77 \mathrm{~K}$ may be assigned to the onset of the structural phase transition being close to first
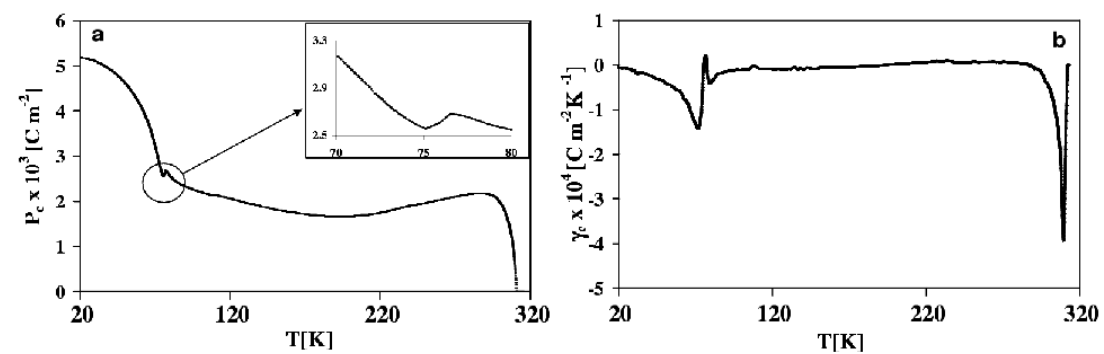

Fig. 5. (a) Spontaneous polarization $\left(P_{\mathrm{s}}\right)$ and (b) the pyroelectric coefficient $(\gamma)$ as a function of temperature for MAPBB along the $c$-axis within 20 and $320 \mathrm{~K}$. The inset in part (a) shows an anomaly of $P_{\mathrm{s}}$ in the vicinity of $77 \mathrm{~K}$. 
order. However, the lower temperature peak of $\gamma_{c}(T)$ curve justifies an important increase in $P_{\mathrm{s}}$ over the lowest temperature phase. It should be also added that quite similar increase in the $P_{\mathrm{s}}$ value is found in $\left(\mathrm{CH}_{3} \mathrm{NH}_{3}\right)_{5} \mathrm{Bi}_{2} \mathrm{Cl}_{11}$ (MAPCB) at $170 \mathrm{~K}$, without any evident presence of the structural phase transition.

One can conclude that the $77 \mathrm{~K}$ phase transition may be explained in terms of the coupling of the order parameter assigned to the structural phase transition with the spontaneous polarization (the $P_{\mathrm{s}}$ value in the lower temperature phase is probably not the order parameter). The type of the transition is classified as an improper ferroelectric phase transition. The suggested coupling of the order parameter with $P_{\mathrm{s}}$ in MAPBB seems to be stronger than that for the chlorine analogue (MAPCB).

The presence of these two possible effects is better visible taking into account the spontaneous polarization evolution with temperature changing along the $a$-axis (see Fig. 6a,b). Since over the intermediate polar phase (from $311.5 \mathrm{~K}$
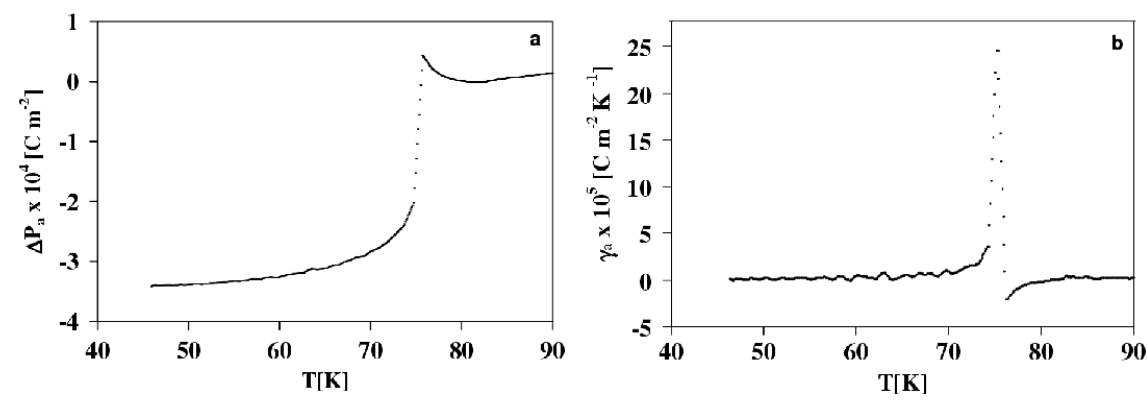

Fig. 6. The same as in Fig. 5 but along the $a$-axis in the vicinity of phase transition at $77 \mathrm{~K}$.

to $77 \mathrm{~K}$ ) the spontaneous polarization component $P_{\mathrm{s}}$ along the $a$-axis should be zero, the observed stepwise change in the $P_{\mathrm{s}}$ value $\left(\Delta P_{\mathrm{s}(a)}\right)$ below $77 \mathrm{~K}$ is strictly connected with the structural phase transition and appearance of a new component of the $P_{\mathrm{s}}$ vector. This phase transition is accompanied by a significant pyroelectric coefficient $\left(\gamma_{a \max }=2.5 \times 10^{-4} \mathrm{C} \mathrm{m}^{-2} \mathrm{~K}^{-1}\right)$.

\section{Conclusions}

1. The dilatometric measurements indicate that the $\left(\mathrm{CH}_{3} \mathrm{NH}_{3}\right)_{5} \mathrm{Bi}_{2} \mathrm{Br}_{11}$ crystals undergo a low temperature ferroelectric transition at $77 \mathrm{~K}$ close to the first order type.

2. The pyroelectric measurements reveal the complex mechanism of the phase transition at $77 \mathrm{~K}$. The low temperature ferroelectric phase transition is believed to be classified as an "improper" one. 


\section{References}

[1] R. Jakubas, L. Sobczyk, Phase Transit. 20, 163 (1990).

[2] V. Varma, R. Bhattacharjee, H.N. Vasan, C.N.R. Rao, Spectrochim. Acta A 48, 1631 (1992).

[3] L. Sobczyk, R. Jakubas, J. Zaleski, Polish J. Chem. 71, 265 (1997) (and references cited therein).

[4] R. Jakubas, Z. Czapla, Z. Galewski, L. Sobczyk, O.J. Żogał, T. Lis, Phys. Status Solidi A 93, 449 (1986).

[5] A. Kallel, J.W. Bats, Acta Crystallogr. C 41, 1022 (1985).

[6] B. Chabot, E. Parthe, Acta Crystallogr. B 34, 645 (1978).

[7] R. Jakubas, U. Krzewska, G. Bator, L. Sobczyk, Ferroelectrics 77, 129 (1988).

[8] R. Jakubas, Solid State Commun. 69, 267 (1989).

[9] R. Jakubas, Z. Czapla Z. Galewski, L. Sobczyk, Ferroelectrics Lett. 5, 143 (1986).

[10] J. Zaleski, A. Pietraszko, Acta Crystallogr. B 52, 287 (1996).

[11] J. Matuszewski, R. Jakubas, T. Głowiak, L. Sobczyk, Acta Crystallogr. C 46, 1385 (1990).

[12] J. Lefebvre, P. Carpentier, R. Jakubas, Acta Crystallogr. B 51, 167 (1995).

[13] J. Jóźków, G. Bator, R. Jakubas, A. Pietraszko, J. Chem. Phys. 114, 7239 (2001).

[14] R. Jakubas, Solid State Commun. 69, 267 (1989).

[15] R. Jakubas, J. Lefebvre, Ferroelectrics 108, 115 (1990).

[16] Cz. Pawlaczyk, H. Motsch, R. Jakubas, H.G. Unruh, Ferroelectrics 108, 127 (1990).

[17] J. Mróz, R. Jakubas, Solid State Commun. 72, 813 (1989).

[18] R. Jakubas, H. Pykacz, Phys. Status Solidi A 115, K17 (1989).

[19] R. Jakubas, P. Francois, J. Lefebvre, Acta Phys. Pol. A 85, 579 (1994).

[20] J. Dziedzic, B. Stolecki, Acta Phys. Pol. A 98, 55 (2000). 